\title{
Article
}

\section{The Prognostic Value of the Lymph Node in Oesophageal Adenocarcinoma; Incorporating Clinicopathological and Immunological Profiling}

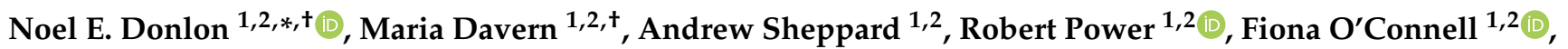 \\ Aisling B. Heeran ${ }^{1,2}$, Ross King ${ }^{1,2} \oplus$, Conall Hayes ${ }^{1,2} \oplus$, Anshul Bhardwaj ${ }^{1}$, James J. Phelan ${ }^{1,2}($, \\ Margaret R. Dunne 1,2 ${ }^{1}$, Narayanasamy Ravi ${ }^{1,2}$, Claire L. Donohoe ${ }^{1,2}$, Jacintha O'Sullivan ${ }^{1,2}$, \\ John V. Reynolds ${ }^{1,2}$ and Joanne Lysaght ${ }^{1,2}$ (i)
}

1 Department of Surgery, Trinity Translational Medicine Institute, Trinity College Dublin, St James's Hospital, D08 W9RT Dublin, Ireland; davernma@tcd.ie (M.D.); SHEPPAA@tcd.ie (A.S.); POWERR8@tcd.ie (R.P.); oconnefi@tcd.ie (F.O.); heerana@tcd.ie (A.B.H.); ROKING@TCD.IE (R.K.); chayes5@tcd.ie (C.H.); Anshul.Bhardwaj@tcd.ie (A.B.); phelanj1@tcd.ie (J.J.P.); dunnem12@tcd.ie (M.R.D.); nravi@stjames.ie (N.R.); donohoe.claire@gmail.com (C.L.D.); osullij4@tcd.ie (J.O.); reynoldsjv@stjames.ie (J.V.R.); jlysaght@tcd.ie (J.L.)

2 Trinity St James's Cancer Institute, St James's Hospital, D08 W9RT Dublin, Ireland

* Correspondence: donlonn@tcd.ie

+ These authors contributed equally to this paper.

Citation: Donlon, N.E.; Davern, M.; Sheppard, A.; Power, R.; O'Connell, F.; Heeran, A.B.; King, R.; Hayes, C.; Bhardwaj, A.; Phelan, J.J.; et al. The Prognostic Value of the Lymph Node in Oesophageal Adenocarcinoma; Incorporating Clinicopathological and Immunological Profiling. Cancers 2021, 13, 4005. https://doi.org/ 10.3390/cancers13164005

Academic Editors: Takashi Ono, Bas P. L. Wijnhoven and Inna Chervoneva

Received: 24 May 2021

Accepted: 4 August 2021

Published: 9 August 2021

Publisher's Note: MDPI stays neutral with regard to jurisdictional claims in published maps and institutional affiliations.

Copyright: (c) 2021 by the authors. Licensee MDPI, Basel, Switzerland. This article is an open access article distributed under the terms and conditions of the Creative Commons Attribution (CC BY) license (https:// creativecommons.org/licenses/by/ $4.0 /)$.
Simple Summary: Oesophageal cancer rates are increasing rapidly with patients often presenting at an advanced stage. The current approach to treatment involves radiotherapy, chemotherapy, or combination chemoradiotherapy with surgery; however, only a fraction of these patients will achieve meaningful responses. Therefore, there is a need to better understand the tumour and lymph node microenvironments to inform future treatment strategies. This study measured immune markers including immune checkpoint expression in tumour and lymph node tissue in oesophageal cancer patients and patient clinical outcomes, including survival time, response to treatment, and adverse events. We report herein that nodal metastases is of equal prognostic importance to clinical tumour stage and tumour regression grade in OAC and we observed a more immunosuppressive microenvironment in the tumour compared with the lymph node.

Abstract: Response rates to the current gold standards of care for treating oesophageal adenocarcinoma (OAC) remain modest with $15-25 \%$ of patients achieving meaningful pathological responses, highlighting the need for novel therapeutic strategies. This study consists of immune, angiogenic, and inflammatory profiling of the tumour microenvironment (TME) and lymph node microenvironment (LNME) in OAC. The prognostic value of nodal involvement and clinicopathological features was compared using a retrospective cohort of OAC patients $(n=702)$. The expression of inhibitory immune checkpoints by $\mathrm{T}$ cells infiltrating tumour-draining lymph nodes (TDLNs) and tumour tissue post-chemo(radio)therapy at surgical resection was assessed by flow cytometry. Nodal metastases is of equal prognostic importance to clinical tumour stage and tumour regression grade (TRG) in OAC. The TME exhibited a greater immuno-suppressive phenotype than the LNME. Our data suggests that blockade of these checkpoints may have a therapeutic rationale for boosting response rates in OAC.

Keywords: oesophageal adenocarcinoma; immune checkpoints; tumour-draining lymph node; prognostic; immunophenotyping; metastatic niche

\section{Introduction}

Multimodal neoadjuvant therapy consisting of chemotherapy or chemoradiotherapy has become the standard-of-care for stage II-III cancer of the oesophagus and the oesophagogastric junction (OGJ). The potential benefits of neoadjuvant therapy include 
downstaging of the primary tumour, facilitating complete surgical resection and eradicating occult micrometastases [1]. However, neoadjuvant treatment results in a complete pathological response in just $15-30 \%$ of patients, and it is this subgroup that gains the survival benefit from systemic therapy [2,3]. It is therefore important to identify those that are at a high risk of recurrence after perioperative therapy as these patients may benefit from alternative regimens. As treatment is associated with substantial morbidity, early identification of non-responders could reduce the toxicity burden of minimally effective systemic therapies.

The lymphatic system has been recognised as a route of metastasis for over 150 years [4-6] and clinical nodal status $(\mathrm{cN})$ is part of the preoperative staging of oesophageal cancer. Curative surgery includes lymph node dissection and [7] pathological staging of resected nodes ( $\mathrm{ypN}$ ) has been shown to independently predict survival for oesophageal cancer patients [8-12]. In addition to nodal status, histological tumour regression following treatment, measured by different tumour regression grade (TRG) scales, can predict overall survival [11-15]. However, in a secondary analysis of a randomised trial, only the nodal status was an independent prognostic factor [16]. The downgrading of nodal status following neoadjuvant treatment (where $\mathrm{ypN}$ is less than $\mathrm{cN}$ ) also positively correlates with survival [17], particularly in patients that do not display a local response in their primary tumour [15]. However, it has been reported that those with nodal downstaging have shorter median survival than node-negative patients before treatment $[17,18]$.

Along with their role in metastasis and as a prognostic factor, tumour-draining lymph nodes (TDLNs) are important in the anti-tumour immune response [19]. Priming of antitumour $\mathrm{CD}^{+} \mathrm{T}$ cells by dendritic cells (DCs) occurs in the lymph node, a critical step in the cancer immunity cycle that is potentiated by anti-CTLA-4 immune checkpoint blockade (ICB) $[20,21]$. However, TDLNs are rich in tumour-derived factors such as IL-6, TGF$\beta$, prostaglandin E2, and VEGF, which promote an immunosuppressive milieu [19]. In melanoma, breast and cervical cancer, the lymph node microenvironment (LNME) promotes an immature and suppressive immune cell phenotype, through increased regulatory $\mathrm{T}$ cell $\left(\mathrm{T}_{\text {reg }}\right)$ and myeloid-derived suppressor cell (MDSC) infiltration as well as by a generalized state of enhanced T cell anergy. In addition, lymph node (LN)-resident DC subsets show lower levels of activation compared with that of migratory DC subsets [22-24], subsequently attenuating $\mathrm{T}$ cell activation, thus allowing for subsequent tumour progression and metastasis [25]. In this altered cytokine milieu, $\mathrm{CD}^{+} \mathrm{T}$ cells do not differentiate into effector $\mathrm{T}$ cells but instead differentiate into peripheral $\mathrm{T}_{\text {reg }}$ cells that restrain anti-tumour immunity [26]. The LNME is emerging as an important substrate for immune checkpoint blockade (ICB); in mouse models, ablation or surgical resection of sentinel lymph nodes reduces immune cell infiltration in the primary tumour and reduced the efficacy of antiPD-1 and anti-PD-L1 therapy [27]. These studies highlight the complex yet critical role of TDLNs in promoting or inhibiting anti-tumour immunity and mediating response to ICB [28]. Furthermore, elective nodal irradiation in mice reduces chemokine expression in the tumour microenvironment (TME) and subsequent intratumoural CD8 $8^{+} \mathrm{T}$ cell infiltration resulting in reduced ICB efficacy [29]. This also has therapeutic implications, as local delivery of ICB to TDLNs in mice had similar efficacy to intratumoral delivery [30]. However, the LNME has yet to be examined in the context of oesophagogastric adenocarcinoma. An understanding of the LNME niche would help inform the development of rational immunotherapeutic strategies to boost response rates to ICB and conventional therapies in oesophagogastric adenocarcinomas [31-33].

In this study, we investigated the prognostic implications of clinical and pathologic nodal status in a large single-centre cohort of patients with locally advanced resectable oesophagogastric cancer. Additionally, we profiled immune checkpoint (IC) expression on T cells residing in the TDLN and infiltrating-tumour tissue of oesophagogastric adenocarcinoma patients. Furthermore, the inflammatory, angiogenic, cytokine, and chemokine profile of the LNME and TME in oesophagogastric cancer patients is investigated and correlated with clinicopathologic outcomes. This information will help provide a fundamental 
understanding of the LNME and TME in oesophagogastric adenocarcinoma patients in order to better inform future therapeutic approaches.

\section{Methods}

\subsection{Ethics Statement}

Ethical approval was granted from Tallaght/St James's Hospital Ethics Committee. Informed written consent was obtained for all the sample and data collection, which was carried out using best clinical practice guidelines. All procedures followed were in accordance with the ethical standards of the responsible committee on human experimentation (institutional and national) and with the Helsinki Declaration of 1975, as revised in 2008. Patient samples were pseudonymised to protect privacy rights.

\subsection{Specimen Collection}

All patients who consented to fresh specimen collection were enrolled between 2018 and 2020. Tumour biopsies $(n=9)$ along with matched TDLN tissue biopsies $(n=6)$ were obtained from oesophageal adenocarcinoma patients at time of surgical resection at $\mathrm{St}$ James's Hospital, Dublin, Ireland. Paraoesophageal lymph nodes were confirmed to be negative for metastatic disease by formal laboratory histological analysis.

\subsection{LN and Tumour Tissue Digestion}

Biopsies were enzymatically digested to perform single-cell phenotyping. Briefly, tissue was minced using a scalpel and digested in collagenase solution $(2 \mathrm{mg} / \mathrm{mL}$ of collagenase type IV (Sigma, MO, USA) in Hanks Balanced Saline Solution (GE healthcare, Wauwatosa, WI, USA) supplemented with $4 \%(v / v)$ foetal bovine serum) at $37^{\circ} \mathrm{C}$ and agitated at $1500 \mathrm{rpm}$ on an orbital shaker. Tissue was then filtered using $70 \mathrm{uM}$ nylon mash filter and washed with FACs buffer (PBS containing 1\% foetal bovine serum and $0.01 \%$ sodium azide). The resulting single cell suspensions were then stained for flow cytometry.

\subsection{Flow Cytometry Staining}

LN and tumour tissue biopsies were stained with zombie aqua viability dye (Biolegend, San Diego, CA, USA) and the following cell surface antibodies: PD-L1 PE and CD8 BV421 (BD Biosciences, Franklin Lakes, NJ, USA), TIM-3 Viobright FITC and CD3 APC (Miltenyi, Bergisch Gladbach, Germany), TIGIT PE/Cy7 and PD-1 APC/Cy7 (Biolegend, San Diego, CA, USA) and CD4 PerCpCy5.5 (eBiosciences, San Diego, CA, USA). Cells were fixed with $1 \%$ paraformaldehyde solution (Santa Cruz, TX, USA), washed and resuspended in FACs buffer, and acquired using BD FACs CANTO II (BD Biosciences) using Diva software vX and analysed using FlowJo v10 software (TreeStar Inc., Ashland, OR, USA).

\subsection{Generation of Lymph Node Conditioned Media (LNCM) and Tumour Conditioned $\operatorname{Media}(T C M)$}

LN $(n=6)$ and tumour tissue $(n=6)$ biopsies were cultured in a 12-well plate in $1 \mathrm{~mL}$ of L-15 (Leibovitz) Lonza ${ }^{\mathrm{TM}}$ BioWhittaker ${ }^{\mathrm{TM}}$ (Basel, Switzerland) X-vivo media for $24 \mathrm{~h}$ ex vivo at $37^{\circ} \mathrm{C}, 5 \% \mathrm{CO}_{2}$. $\mathrm{LN}$ tissue was divided into four equal quadrants to ensure adequate distribution of immune cells. LN were confirmed to be benign through formal histological assessment by the St James' Hospital Histopathology department. The resulting lymphnode-conditioned media (LNCM) and tumour-conditioned media (TCM) was harvested and stored at $-80{ }^{\circ} \mathrm{C}$ until required for further experimentation.

\subsection{Quantification of Serum Immune Proteins}

A panel of 54 angiogenic, vascular injury, pro-inflammatory, cytokine, and chemokine mediators were quantified by 54-plex ELISA in LNCM and TCM according to the manufacturer's instructions (Meso Scale Diagnostics, Rockville, MA, USA). The following secretions were quantified in the LNCM and TCM: CRP, Eotaxin, Eotaxin-3, bFGF, Flt-1, GM-CSF, ICAM-1, IFN- $\gamma$, IL-10, IL-12/IL-23p40, IL-12p70, IL-13, IL-15, IL-16, IL-17A, IL-17A/F, 
IL-17B, IL-17C, IL-17D, IL-1RA, IL-1 $\alpha$, IL-1 $\beta$, IL-2, IL-21, IL-22, IL-23, IL-27, IL-3, IL-31, IL-4, IL-5, IL-6, IL-7, IL-8, IL-8 (High Abundance), IL-9, IP-10, MCP-1, MCP-4, MDC, MIP$1 \alpha$, MIP- $1 \beta$, MIP-3 $\alpha$, PIGF, SAA, TARC, Tie- 2, TNF- $\alpha$, TNF- $\beta$, TSLP, VCAM- 1, VEGF-A, VEGF-C and VEGF-D. All assays were run as per the manufacturer's instructions, with an overnight supernatant incubation protocol used for all assays except angiogenesis panel 1 and vascular injury panel 2 , which were run according to the same day protocol. TCM and LNCM were run undiluted on all assays except vascular injury panel 2, where a one in four dilution was used, as per previous optimisation experiments. Secretion data for all factors was normalised to cell lysate protein content determined using a BCA assay (Pierce, Waltham, MA, USA).

\subsection{Statistical Analysis}

Statistical analysis was performed using SPSS ${ }^{\circledR}$ (version 18.0) software (SPSS, Chicago, IL, USA) and R 2.13.2. A significance level of $p<0.05$ was used for all analyses and all $p$ values reported are two-tailed. The Kaplan-Meier method and the log rank test was used to assess differences in survival between groups. Survival time was measured from the date of first treatment to the date of death or last follow-up. Independent variables were entered into a multivariable Cox proportional hazards model, variables found at univariable analysis to have a $p$ value $<0.05$ were entered into the multivariable model. Continuous variables were compared using unpaired $t$-tests. Association of categorical variables was assessed using $\chi^{2}$ test.

Wilcoxon rank test was utilized to compare checkpoint, cytokine, and markers of angiogenesis expression between the lymph node and the tumour compartment.

\section{Results}

3.1. Nodal Status and Pathological Complete Response Are Equally Important Prognostication Markers in OAC in Predicting Overall Survival Time

Data for 702 patients were obtained from a prospectively maintained database between 2001 and 2016, of these 356 were classified as node negative, with a higher proportion of the N0 cohort having a Siewert type I tumour (60\%). An en bloc radical oesophagectomy was performed in $50 \%$ of N0,66\% N1, 48\% of N2, and 53\% of N3 (Table 1). On pathologic assessment of resected specimens, N0 had earlier T stage disease, with $54 \%$ being (y)p or pT0/T1, compared with $18 \%$ for N1, $9 \%$ for $\mathrm{N} 2$, and $7 \%$ for N3 $(p<0.001)$. N0 and N1 were significantly associated with Barrett's oesophagus, 55\% and 49\%, respectively $(p<0.001)$, with signet ring and mucinous features more frequent in N2 and N3. N0 was associated with less adverse features of tumour when compared to those with nodal positivity. Adverse features consisted of poor differentiation, perineural, lymphatic, and vascular invasion, with a novel three-grouping stratification of $0,1-2$, and $3-4$ adverse features previously described by our department [34]. Linking adverse pathology with nodal status, $52 \%, 42 \%$, and $6 \%$ of N0 were in the $0,1-2$, and 3-4 group, respectively, compared with $16 \%, 58 \%$, and $26 \%$ for N1; $6 \%, 51 \%$, and $43 \%$ for N2; and $5 \%, 44 \%$, and $51 \%$ for N3 $(p<0.001)$. Chemoradiation was the most commonly utilised neoadjuvant treatment modality across all nodal status. Mandard TRG after neoadjuvant therapy was significantly better in N0, with $25 \%$ either TRG 1 or 2 , compared with $14 \%$ in N1, $12 \%$ in $\mathrm{N} 2$, and $8 \%$ in N3. The clinical and pathologic demographics per nodal burden are outlined in Table 1. 
Table 1. Clinical characteristics for patient cohort included in study.

\begin{tabular}{|c|c|c|c|c|c|}
\hline Clinical Characteristics & pNo & pN1 & $\mathrm{pN} 2$ & $\mathrm{pN} 3$ & \\
\hline & $N=356$ & $N=154$ & $N=47$ & $N=145$ & $p$-value \\
\hline Age, mean (SD) & $63.62(10.47)$ & $62.97(10.03)$ & $65.02(10.09)$ & $63.22(10.85)$ & 0.64 \\
\hline \multicolumn{6}{|l|}{ Sex, $(n)$} \\
\hline Female & 62 & 24 & 6 & 28 & \\
\hline Male & 294 & 130 & 41 & 117 & 0.71 \\
\hline BMI, kg/m ${ }^{-2}$, mean (SD) & $27.47(4.58)$ & $27.32(4.35)$ & $28.65(4.62)$ & $26.81(4.54)$ & 0.78 \\
\hline Obese & 218 & 97 & 33 & 74 & 0.15 \\
\hline Current smoker & 156 & 6 & 18 & 49 & 0.38 \\
\hline Diabetes & 4 & 18 & 8 & 16 & 0.74 \\
\hline Hypertension & 106 & 40 & 9 & 39 & 0.43 \\
\hline Dyslipidemia & 62 & 20 & 9 & 12 & 0.046 \\
\hline \multicolumn{6}{|l|}{ ASA grade, } \\
\hline ASA I & 139 & 57 & 13 & 51 & \\
\hline ASA II & 166 & 78 & 23 & 77 & \\
\hline ASA III & 51 & 19 & 11 & 17 & 0.37 \\
\hline \multicolumn{6}{|l|}{ Siewert Classification } \\
\hline Siewert I & 217 & 76 & 19 & 48 & \\
\hline Siewert II & 77 & 48 & 11 & 39 & \\
\hline Siewert III & 62 & 30 & 17 & 58 & $<0.001$ \\
\hline \multicolumn{6}{|l|}{ Treatment characteristics } \\
\hline \multicolumn{6}{|l|}{ Treatment pathway: } \\
\hline Neoadjuvant therapy & 186 & 106 & 24 & 79 & \\
\hline Surgery first & 170 & 48 & 23 & 66 & 0.005 \\
\hline \multicolumn{6}{|l|}{ Neoadjuvant regimen: } \\
\hline Chemotherapy & 59 & 39 & 7 & 39 & \\
\hline Chemoradiation & 127 & 67 & 17 & 40 & 0.04 \\
\hline \multicolumn{6}{|l|}{ Operation type } \\
\hline 2-stage esophagectomy & 197 & 101 & 23 & 77 & \\
\hline 3-stage esophagectomy & 27 & 10 & 0 & 15 & \\
\hline Transhiatal esophagectomy & 78 & 12 & 7 & 7 & $<0.001$ \\
\hline Extended total gastrectomy & 54 & 31 & 17 & 46 & \\
\hline \multicolumn{6}{|l|}{ Clinical and Pathologic } \\
\hline \multicolumn{6}{|l|}{ Clinical T stage } \\
\hline 1 & 112 & 14 & 2 & 4 & \\
\hline 2 & 58 & 21 & 6 & 19 & \\
\hline 3 & 183 & 114 & 38 & 114 & \\
\hline 4 & 3 & 5 & 1 & 8 & $<0.001$ \\
\hline
\end{tabular}


Table 1. Cont.

\begin{tabular}{|c|c|c|c|c|c|}
\hline Clinical Characteristics & pNo & pN1 & $\mathrm{pN} 2$ & $\mathrm{pN} 3$ & م Vlo \\
\hline & $N=356$ & $N=154$ & $N=47$ & $N=145$ & $p$-value \\
\hline \multicolumn{6}{|l|}{ Clinical N stage } \\
\hline 0 & 256 & 87 & 28 & 57 & \\
\hline 1 & 87 & 57 & 15 & 73 & \\
\hline 2 & 12 & 9 & 3 & 11 & \\
\hline 3 & 1 & 1 & 1 & 4 & $<0.001$ \\
\hline Proximal Margin Clear & 353 & 149 & 46 & 133 & $<0.001$ \\
\hline Distal Margin Clear & 353 & 146 & 44 & 131 & $<0.001$ \\
\hline Radial Margin Clear & 305 & 112 & 36 & 71 & $<0.001$ \\
\hline Lymphatic invasion & 51 & 100 & 35 & 119 & $<0.001$ \\
\hline Venous invasion & 65 & 66 & 27 & 92 & $<0.001$ \\
\hline Perineural invasion & 25 & 35 & 16 & 50 & $<0.001$ \\
\hline $\begin{array}{l}\text { Poor or undifferentiated grade, } \\
\text { (poor versus other) }\end{array}$ & 110 & 59 & 23 & 84 & $<0.001$ \\
\hline Barrett's oesophagus & 173 & 58 & 17 & 34 & $<0.001$ \\
\hline Signet Ring & 25 & 13 & 4 & 32 & $<0.001$ \\
\hline Mucinous features & 33 & 18 & 6 & 34 & $<0.001$ \\
\hline Barrett's in Tumour & 194 & 75 & 18 & 47 & $<0.001$ \\
\hline \multicolumn{6}{|l|}{ Adverse feature grading: } \\
\hline No adverse features & 184 & 25 & 3 & 7 & $<0.001$ \\
\hline 1 or 2 adverse features & 151 & 90 & 24 & 64 & \\
\hline 3 or 4 adverse features & 21 & 39 & 20 & 74 & \\
\hline \multicolumn{6}{|l|}{ Pathologic stage: } \\
\hline (y)pT0 & 37 & 1 & 1 & 3 & \\
\hline (y)pT1 & 154 & 26 & 3 & 7 & \\
\hline (y)pT2 & 67 & 29 & 7 & 14 & $<0.001$ \\
\hline (y)pT3 & 92 & 87 & 34 & 105 & \\
\hline (y)pT4 & 6 & 11 & 2 & 16 & \\
\hline \multicolumn{6}{|l|}{ Tumour regression grade N (\%) } \\
\hline TRG 1 & 40 & 1 & 1 & 1 & \\
\hline TRG 2 & 51 & 20 & 5 & 12 & \\
\hline TRG 3 & 54 & 40 & 7 & 19 & \\
\hline TRG 4 & 33 & 33 & 8 & 30 & \\
\hline TRG 5 & 9 & 13 & 4 & 17 & $<0.001$ \\
\hline \multicolumn{6}{|l|}{ CAP } \\
\hline R0 & 335 & 133 & 37 & 95 & \\
\hline $\mathrm{R} 1$ & 21 & 21 & 10 & 50 & $<0.001$ \\
\hline \multicolumn{6}{|l|}{ RCPATH } \\
\hline R0 & 302 & 109 & 34 & 79 & \\
\hline $\mathrm{R} 1$ & 54 & 45 & 13 & 66 & $<0.001$ \\
\hline
\end{tabular}


Table 1. Cont.

\begin{tabular}{|c|c|c|c|c|c|}
\hline Clinical Characteristics & pNo & $\mathrm{pN1}$ & $\mathrm{pN} 2$ & $\mathrm{pN} 3$ & -Valuo \\
\hline & $N=356$ & $N=154$ & $N=47$ & $N=145$ & $p$-varue \\
\hline Recurrence & 97 & 83 & 29 & 115 & $<0.001$ \\
\hline Median Survival (Months) & Not reached & 49.7 & 34.23 & 15.43 & $<0.001$ \\
\hline 1 Year Survival & 94 & 86 & 83 & 67 & \\
\hline 3 Year Survival & 78 & 54 & 48 & 21 & $<0.001$ \\
\hline 5 Year Survival & 67 & 45 & 38 & 11 & \\
\hline
\end{tabular}

Adverse features of tumour biology including perineural invasion, vascular invasion, differentiation nodal positivity, and neoadjuvant treatment were significant by multivariate analysis on overall survival. (Table 2).

Table 2. Univariable and multivariable analysis for overall survival.

\begin{tabular}{ccccccc}
\hline Variable & & Univariable & & \multicolumn{2}{c}{ Multivariable } \\
\hline & HR & CI & $p$ Value & HR & CI & $p$ Value \\
\hline Barretts history & 1.44 & $1.138-1.821$ & $<0.001$ & 1.680 & $0.919-3.072$ & 0.09 \\
\hline Neoadjuvant treatment & 1.528 & $1.211-1.928$ & $<0.001$ & 1.434 & $1.177-1.746$ & $<0.001$ \\
\hline $\mathrm{pN}$ (node negative vs. positive) & 1.577 & $1.104-1.747$ & $<0.001$ & 1.399 & $1.151-1.703$ & $<0.001$ \\
\hline Perineural invasion & 1.601 & $1.257-2.039$ & $<0.001$ & 1.475 & $1.125-1.935$ & 0.005 \\
\hline Vascular invasion & 1.548 & $1.219-1.465$ & $<0.001$ & 0.527 & $0.316-0.879$ & 0.01 \\
\hline Differentiation (poor versus other) & 1.584 & $1.245-2.017$ & $<0.001$ & 1.582 & $1.098-1.595$ & 0.02 \\
\hline
\end{tabular}

For the entire cohort classified by nodal status (Figure 1), the median overall survival was not reached for N0, 49.7 months for N1, 34.2 months for N2, and 15.4 months for N3 with 5 -year survival of $67 \%, 45 \%, 38 \%$, and $11 \%$, respectively $(p<0.001)$. The median overall survival was 58.27 months (CI 46.28-70.26). The five year survival for node negative disease in $67 \%, 45 \%$ for $\mathrm{N} 1$ disease, $38 \%$ for N2, and $11 \%$ for N3 disease (Table 3).

Table 3. Overall number of surviving OAC patients based on nodal status (pathological post-resection).

\begin{tabular}{|c|c|c|c|c|c|c|c|c|c|c|c|c|}
\hline \multirow{2}{*}{$\begin{array}{c}\text { Survival } \\
\text { (Years) }\end{array}$} & \multicolumn{3}{|c|}{ No } & \multicolumn{3}{|c|}{ N1 } & \multicolumn{3}{|c|}{ N2 } & \multicolumn{3}{|c|}{ N3 } \\
\hline & $\begin{array}{l}\text { No at } \\
\text { Risk }\end{array}$ & Deaths & $\%$ Survival & $\begin{array}{l}\text { No at } \\
\text { Risk }\end{array}$ & Deaths & $\%$ Survival & $\begin{array}{l}\text { No at } \\
\text { Risk }\end{array}$ & Deaths & \% Survival & $\begin{array}{l}\text { No at } \\
\text { Risk }\end{array}$ & Deaths & $\%$ Survival \\
\hline 1 & 356 & 22 & 94 & 154 & 21 & 86 & 47 & 8 & 83 & 145 & 46 & 67 \\
\hline 3 & 317 & 49 & 78 & 124 & 43 & 54 & 38 & 14 & 48 & 89 & 58 & 21 \\
\hline 5 & 225 & 28 & 67 & 67 & 11 & 45 & 15 & 3 & 38 & 22 & 10 & 11 \\
\hline
\end{tabular}




\section{Survival proportions based on nodal status}

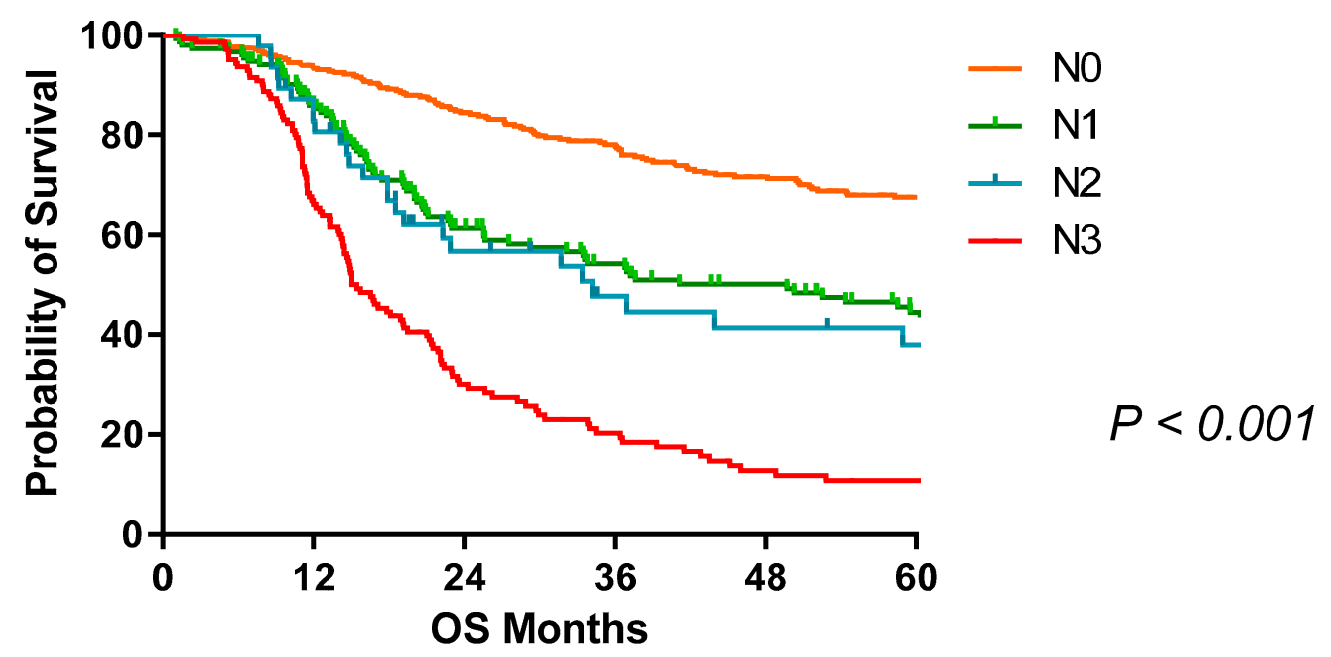

Figure 1. Survival proportions of OAC patients based on nodal status ( $N 0=$ node negative, $\mathrm{N} 1=1-2$ Nodes, N2 = 3-6 nodes, N3 $\geq 7$ ). Any nodal disease burden significantly reduces overall survival with proportional reduction per stage of dissemination. Median survival: N0, not reached; N1, 49.7 months; N2, 34.23 months; N3, 15.43 months.

Patients with positive clinical nodal status (based on endoscopic ultrasound and/or positron emission tomography (PET)) but pathologically node-negative had improved survival compared to those who were clinically and pathologically node-positive $(p<0.001)$ (Figure 2). Important caveats to this finding include the fact that clinical staging is carried out at time of diagnosis and pathological staging is carried out post-surgical resection. There are a multitude of reasons for such discrepancies such as an increase in tumour burden as a consequence of adverse biology or treatment refractory disease reducing responses to neoadjuvant therapy. Similarly, clinical staging through endoscopy, conventional CT TAP and PET/CT imaging cannot detect microscopic disease; therefore, final pathological grading is definitive.

\section{Survival proportions based on ClinicoPathological Nodal Status}

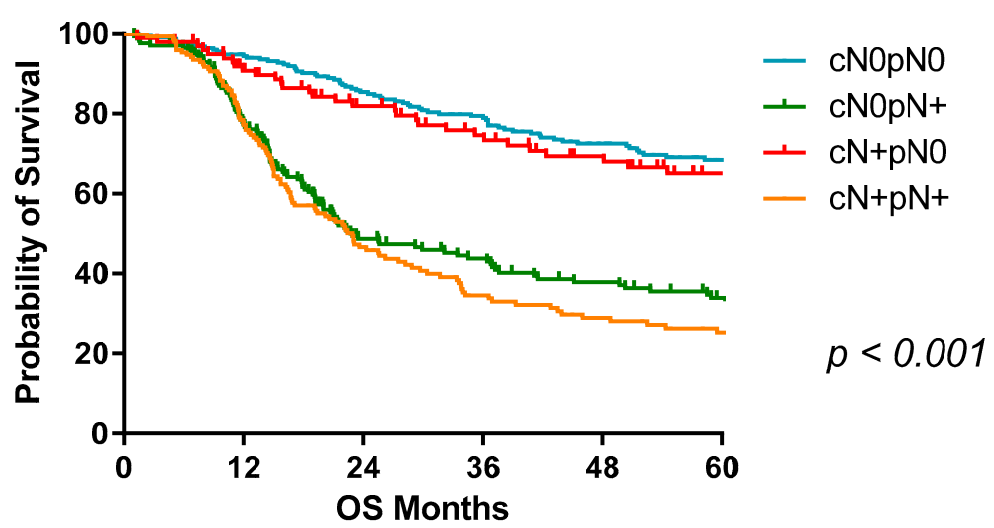

Figure 2. Survival proportions of OAC patients based on clinicopathological nodal status. Median survival: cN0pN0, not reached; $\mathrm{cN}+\mathrm{pN} 0,124.6$ months; $\mathrm{cN} 0 \mathrm{pN}+, 22.93 ; \mathrm{cN}+\mathrm{pN}+, 22.5$ months.

The five-year survival for clinically node negative, pathologically node negative disease is $68 \%$, compared to $61 \%$ in those with clinically node positive, pathologically node negative disease. In those with clinically and pathologically node positive disease, the five-year survival is $26 \%$ (Table 4 ). 
Table 4. Overall survival of OAC patients based on clinicopathological nodal status.

\begin{tabular}{ccccccccccccc}
\hline Survival & \multicolumn{3}{c}{ cN0pN0 } & \multicolumn{3}{c}{ cN+pN0 } & \multicolumn{3}{c}{ cN0pN+ } & \multicolumn{2}{c}{$+\mathrm{pN}+$} \\
\hline (Years) & $\begin{array}{l}\text { No at } \\
\text { Risk }\end{array}$ & Deaths & \% Survival & $\begin{array}{c}\text { No at } \\
\text { Risk }\end{array}$ & Deaths & \% Survival & $\begin{array}{c}\text { No at } \\
\text { Risk }\end{array}$ & Deaths & \% Survival & $\begin{array}{c}\text { No at } \\
\text { Risk }\end{array}$ & Deaths & \% Survival \\
\hline 1 & 256 & 13 & 95 & 100 & 9 & 91 & 172 & 38 & 77 & 174 & 37 & 78 \\
\hline 3 & 231 & 35 & 79 & 86 & 14 & 75 & 126 & 52 & 44 & 125 & 63 & 35 \\
\hline 5 & 166 & 21 & 68 & 59 & 7 & 65 & 61 & 13 & 34 & 43 & 11 & 26 \\
\hline
\end{tabular}

In patients treated with neoadjuvant chemotherapy, the median survival in patients who were pN0 was 79.2 months compared with 71.64 months in those treated with chemoradiotherapy. On the contrary, in patients who were $\mathrm{pN}+$ treated with neoadjuvant chemotherapy, the median survival was 22.9 months compared to 29.73 months in those treated with chemoradiotherapy (Figure 3). Interestingly, patients who were node negative had a higher median survival than those with a PCR on final histological analysis to neoadjuvant therapy and significantly better than those who had nodal positivity $(p<0.001)$ (Figure 4). The 5-year overall survival for those with node positive disease and treated with chemotherapy was $37 \%$ compared with $25 \%$ with chemoradiotherapy (Table 5).

\section{Survival proportions based on Nodal Status and Chemoradiotherapy/Chemotherapy}

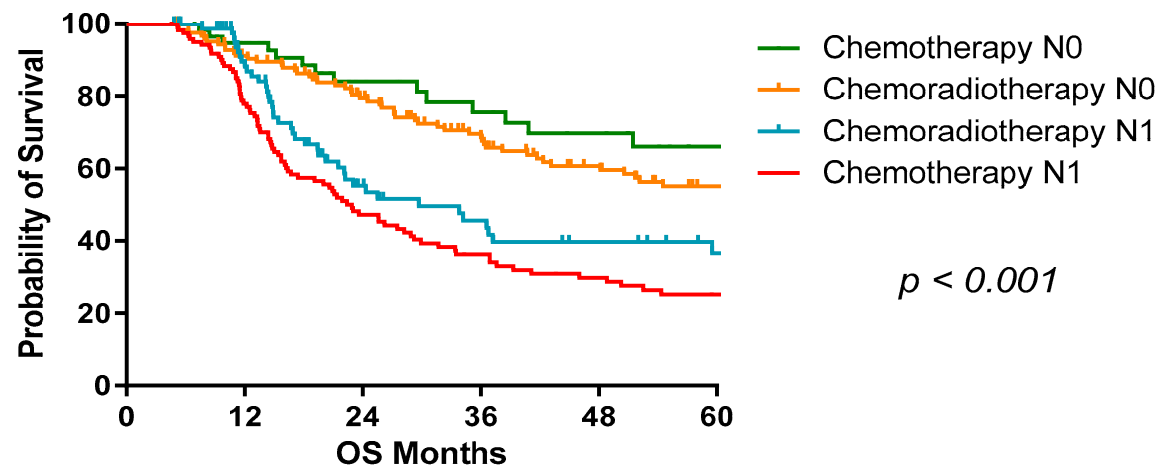

Figure 3. Survival proportions of OAC patients based on nodal status and treatment received (chemoradiotherapy/chemotherapy). Median survival: pN0 chemotherapy, 79.2 months; pN0 chemoradiotherapy, 71.64 months; $\mathrm{pN}+$ chemotherapy, 22.9 months; $\mathrm{pN}+$ chemoradiotherapy, 29.73 months.

\section{Survival proportions based on PCR \& Nodal Status}

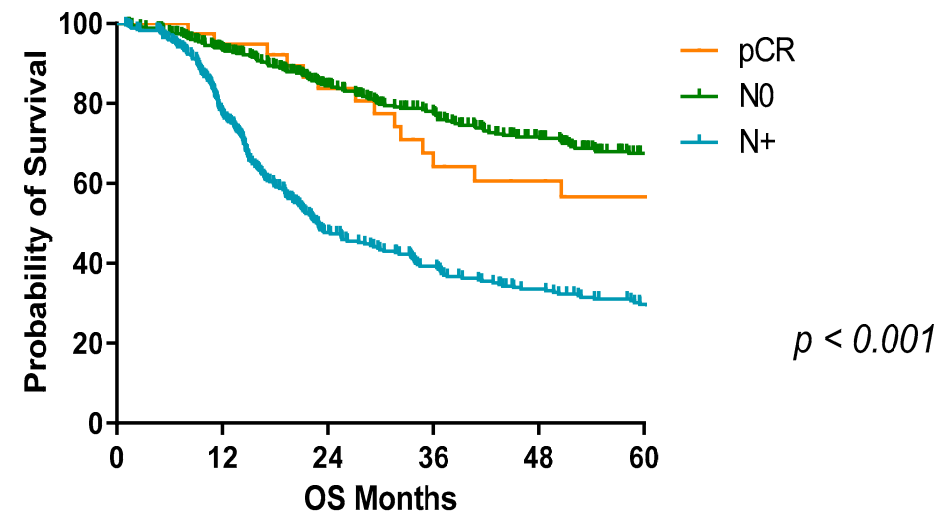

Figure 4. Survival proportions of OAC patients based on nodal status and pathologic complete response. pCR refers to complete pathological response on histological analysis, N0-node negative, $\mathrm{N}+$-node positive. Significantly reduced overall survival with nodal disease. Median survival: $\mathrm{pCR}$, not reached; N0, 124.6 months; $\mathrm{N}+, 22.9$ months. 
Table 5. Overall survival of OAC patients based on nodal status and treatment received (chemoradiotherapy/chemotherapy).

\begin{tabular}{cccccccccccccc}
\hline Survival & \multicolumn{3}{c}{ Chemotherapy N0 } & \multicolumn{3}{c}{ Chemoradiotherapy No } & \multicolumn{2}{c}{ Chemotherapy N+ } & \multicolumn{2}{c}{ Chemoradiotherapy N+ } \\
\hline (Years) & $\begin{array}{c}\text { No at } \\
\text { Risk }\end{array}$ & Deaths & $\%$ Survival & $\begin{array}{c}\text { No at } \\
\text { Risk }\end{array}$ & Deaths & $\%$ Survival & $\begin{array}{c}\text { No at } \\
\text { Risk }\end{array}$ & Deaths & \% Survival & $\begin{array}{c}\text { No at } \\
\text { Risk }\end{array}$ & Deaths & \% Survival \\
\hline 1 & 59 & 3 & 95 & 127 & 11 & 91 & 85 & 8 & 90 & 124 & 26 & 78 \\
\hline 3 & 50 & 8 & 76 & 112 & 25 & 70 & 66 & 28 & 46 & 89 & 45 & 36 \\
\hline 5 & 27 & 3 & 66 & 73 & 14 & 55 & 23 & 4 & 37 & 34 & 10 \\
\hline
\end{tabular}

3.2. Inhibitory Immune Checkpoint Receptors and Ligands Are Expressed at Significantly Higher Levels on Tumour-Infiltrating T Cells Compared to Tumour-Draining Lymph Nodes

Inhibitory ICs play key roles in restraining anti-tumour immunity and with the recent approval of two anti-PD-1 inhibitors, pembrolizumab and nivolumab, for treating oesophagogastric adenocarcinomas, it is important we develop a deeper understanding of IC expression profiles in adenocarcinoma patients. We profiled IC expression on T cells infiltrating OAC tumour tissue and in the TDLN to determine the level of target expression in multimodal therapy.

Overall, a higher percentage of $\mathrm{T}$ cells expressing inhibitory ICs was found within tumour tissues compared to TDLNs and this pattern was consistent across $\mathrm{CD}^{+} \mathrm{T}$ helper and $\mathrm{CD}^{+}$cytotoxic $\mathrm{T}$ cell compartments (Figure 5). A significantly higher percentage of $\mathrm{CD}^{+} \mathrm{CD}^{+} \mathrm{PD}-1^{+}$cells were found in tumour tissue compared with TDLNs (median 18.2 (range $7.41-46.49$ ) vs. 5.52 (range $0.75-13.65), p<0.05$ ). There was also a significantly higher percentage of $\mathrm{CD}^{+}{ }^{\mathrm{TIM}}-3^{+}$(median 7.1 (range 5.23-15.4) vs. 1.33 (range $0.06-5.28)$ ), $\mathrm{CD}^{+} \mathrm{CD}^{+}{ }^{+} \mathrm{TIM}^{-} 3^{+}$(median 7.51 (range $0.35-20.3$ ) vs. 0.36 (range $\left.0.05-2.41\right)$ ) and $\mathrm{CD}^{+} \mathrm{CD}^{+}{ }^{+} \mathrm{TIM}^{-} 3^{+}$(median 6.37 (range $2.31-31.9$ ) vs. 1.3 (range $0.06-4.07$ )) intratumourally compared to the lymph node $(p<0.05)$. There was a significantly higher expression of $\mathrm{CD}^{+}$ PD-L1 ${ }^{+}$cells (median 12.95 (range 2.19-37)) in the tumour compared to (median 2.07 (range $0.15-8.46)$ ) in the lymph node $(p<0.05)$. The expression of $\mathrm{CD}^{+}{ }^{+} \mathrm{PD}-1^{+} \mathrm{TIM}-3^{+}$(median 5.91 (range $0.01-10.39$ ) vs. 0.27 (range $0-0.63$ ), $p<0.05$ ), and $\mathrm{CD}^{+} \mathrm{CD}^{+}{ }^{+} \mathrm{PD}-1^{+} \mathrm{TIM}-3^{+}$(median 3.41 (range $0-10.3$ ) vs. 0.33 (range $0-1.6$ ), $p<0.05$ ), was significantly higher in the tumour than in the lymph node (Figure 5). There was a significantly higher expression of $\mathrm{CD}^{+}$ $\mathrm{CD} 4^{+} \mathrm{PD}-1^{+} \mathrm{PD}-\mathrm{L} 1^{+}$cells intratumourally (median 4.35 (range $0.2-14.18$ ) compared to the lymph node (median 0.33 (range $0-1.6) p<0.05$ ). Similarly, $\mathrm{CD}^{+} \mathrm{CD}^{+}{ }^{+} \mathrm{PD}-1^{+} \mathrm{PD}-\mathrm{L}^{+}{ }^{+}$cells were also found at a significantly higher frequency in tumour-infiltrating tissue compared with TDLNs (median 1.62 (range $0.02-34.6$ ) vs. 0.12 (range 0-0.87) $p<0.05$ ), similarly for $\mathrm{CD}^{+} \mathrm{CD}^{+}{ }^{+} \mathrm{TIM}-3^{+} \mathrm{PD}^{-L} 1^{+}$cells (median 5.03 (range $0.02-42.8$ ) vs. 0.11 (range $0-0.41$ ), $p<0.05$ ) (Figure 5). There was also a significantly higher expression intratumourally of $\mathrm{CD}^{+} \mathrm{PD}^{+}{ }^{+} \mathrm{TIGIT}^{+} \mathrm{TIM}^{+}{ }^{+}$(median $5.63(0.02-7.58)$ vs. $0.03(0-0.09)$ as well as $\mathrm{CD}^{+}$ $\mathrm{PD}^{+}{ }^{+} \mathrm{TIGIT}^{+} \mathrm{PD}^{-L} 1^{+}$(median 6.02 (range 0.42-7.99) vs. 1.93 (range 0.01-4.44) $(p<0.05$ ). Significantly higher frequencies of $\mathrm{CD} 3^{+} \mathrm{CD} 4^{+} \mathrm{PD}-1^{+} \mathrm{TIM}-3^{+} \mathrm{PD}-\mathrm{L} 1^{+}$cells intratumorally (median 0.68 (range $0.01-10.6$ ) vs. 0.03 (range $0-5.65$ ), $p<0.05$ ), (Figure 5). 
Cancers 2021, 13, 4005

11 of 17

PD-1

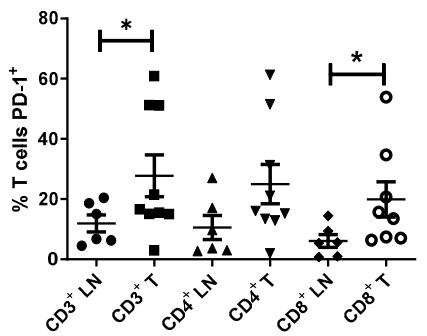

PD-L1

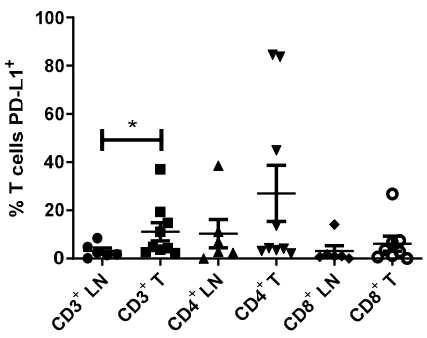

PD -1 ${ }^{+}$PD -Li ${ }^{+}$

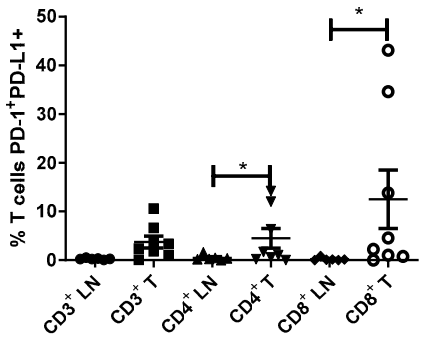

TIM $-3^{+}$PD $-1^{+}$

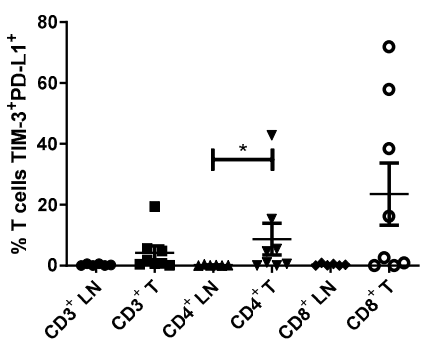

PD -1 ${ }^{+}{ }^{+} I M-3^{+}$PD -Li ${ }^{+}$

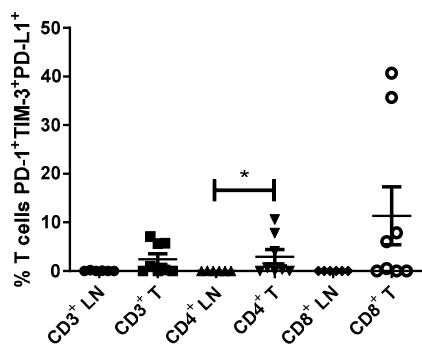

DIGIT

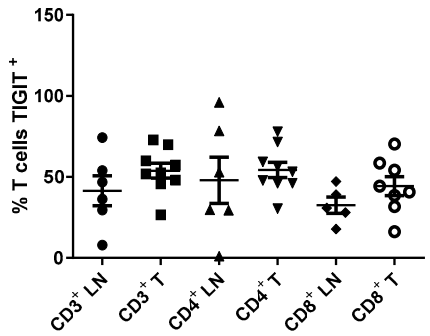

PD -1 ${ }^{+}$TIGIT $^{+}$

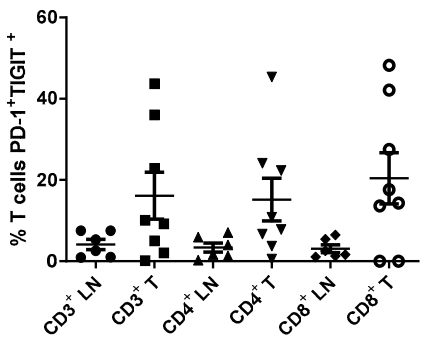

dIGIT ${ }^{+}$TIM -3 ${ }^{+}$

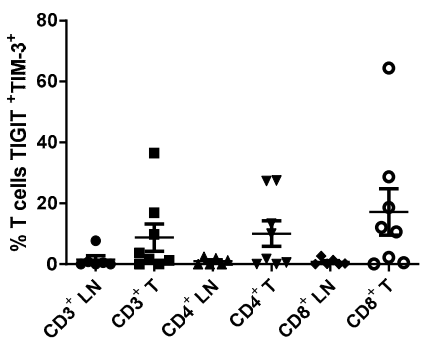

PD $-1^{+}$TIGIT $^{+}$TIM $^{+3^{+}}$

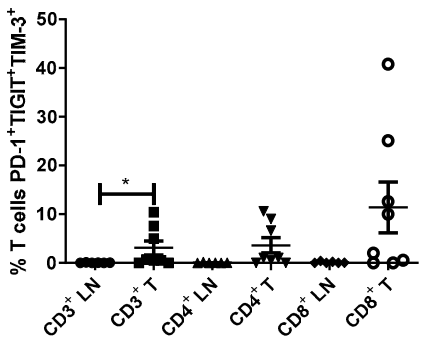

TIM-3

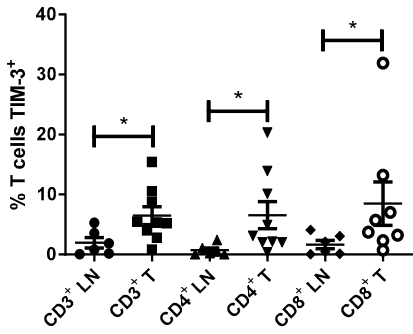

PD -1 ${ }^{+}$TIM $-3^{+}$

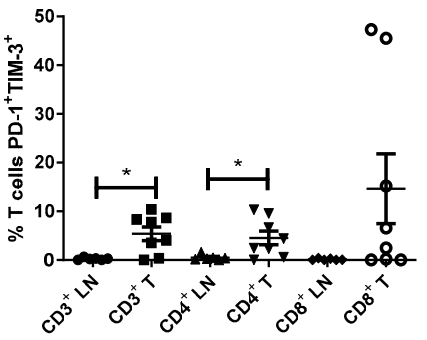

dIGIT $^{+}$PD -LI ${ }^{+}$

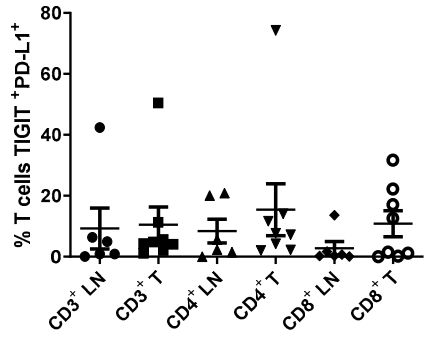

PD-1 ${ }^{+}$TIGIT $^{+}$PD -Li ${ }^{+}$

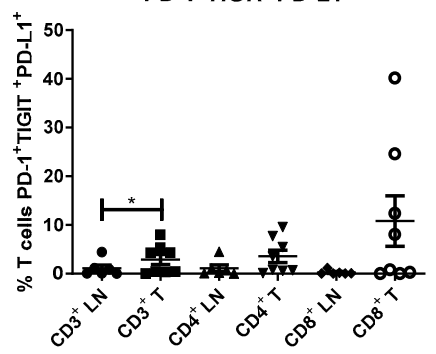

Figure 5. Inhibitory IC receptors and ligands are expressed at significantly higher levels on tumour-infiltrating $\mathrm{T}$ cells compared to $\mathrm{T}$ cells present in tumour-draining lymph nodes. $\mathrm{CD}^{+}, \mathrm{CD}^{+} \mathrm{CD}^{+}, \mathrm{CD}^{+} \mathrm{CD}^{+}$cells in tumour-draining lymph nodes $(n=6)$ and infiltrating tumour tissues $(n=6)$ in OAC patients were screened for the surface expression of PD- $1^{+}, \mathrm{TIGIT}^{+}, \mathrm{TIM}^{+}{ }^{+}$and PD-L1 ${ }^{+}$ex vive by flow cytometric analysis. Tumour draining lymph nodes and tumour specimens were post-treatment and taken at surgical resection. Wilcoxon rank test was used to compare expression between lymph node and tumour compartment; ${ }^{*} p<0.05$. LN: lymph node and T: tumour. 
The levels of anti-tumour cytokines IFN- $\gamma$ were lower in the TME (median $13.63 \mathrm{pg} / \mathrm{mL}$ (range 1.15-43.83) compared to the LNME (median $41.27 \mathrm{pg} / \mathrm{mL}$ (range 18.1-182.2) but did not achieve significance by multivariate analysis (Figure S1). However, the levels of IL-9 and IL-27 cytokines were higher within the TME (median $6.55 \mathrm{pg} / \mathrm{mL}$ (range 2.14-9.86) and $25.92 \mathrm{pg} / \mathrm{mL}$ (range 17.18-337.2)) compared to the LNME (1.31 pg/mL (range 0.41-4.82) and $12.88 \mathrm{pg} / \mathrm{mL}$ (range $0.77-257$ ), respectively, of OAC patients, but again did not achieve significance by multivariate analysis (Figure S1).

The LNME and TME was assessed for pro-angiogenic and vascular damage analytes and the levels of soluble bFGF were higher within the TME $124.8 \mathrm{pg} / \mathrm{mL}$ (range 66.06-610.8) compared with the LNME (median $34.97 \mathrm{pg} / \mathrm{mL}$ (range 22.35-72.94) but were not significant. Interestingly, PIGF was higher within the LNME $116.3 \mathrm{pg} / \mathrm{mL}$ (range 18.78-162.6) than the TME $10.15 \mathrm{pg} / \mathrm{mL}$ (range 4.61-82.11) of OAC patients but was not significant. The levels of all other analytes were detected at comparable levels between the LNCM and TCM from OAC patients, and these cytokines, chemokines, and markers of vascular injury and angiogenesis are available as Table S1.

\section{Discussion}

While there are limitations to the current study, primarily the cohort size of fresh patient samples, this should be borne in mind when considering the conclusions of the study, as this limits the ability to perform clinical correlative work. We have demonstrated in a very large cohort of OAC patients $(n=702)$ that the presence of nodal metastases has equivalent prognostic value to that of clinical tumour stage and TRG in OAC patients. This further highlights that the TDLN may play a pivotal role in orchestrating anti-tumour responses and subsequent treatment response in OAC patients and complements previous studies.

Identifying patients at risk of relapse following surgery for OAC remains a challenge, but histological assessment of surgical specimens is an attractive means of predicting response. TRG 1-2, as determined by the Mandard or Becker systems, has a robust association with overall survival (OS) and disease-free survival in resected specimens [11-15]. The pathological tumour extent in the resected specimen $(\mathrm{pT})$ is also associated with recurrence and survival in OAC. Our results are in agreement with this, as TRG and pT stage are significant prognostic markers in patients following neoadjuvant treatment. In a post hoc analysis of the MAGIC trial of perioperative chemotherapy in resectable oesophagogastric cancer, both TRG1-2 and nodal status were negatively related to survival [16]. However, after multivariate adjustment, it was only the presence of lymph node metastasis that was an independent predictor of OS (HR: 3.36; 95\% CI, 1.70 to 6.63). Interestingly, a machine learning model developed to predict the risk of recurrence following neoadjuvant treatment found that the number of lymph node metastases was the most important variable in the model, with lymphovascular invasion second [35]. This is similar to our findings as we demonstrate that nodal status has equivalent prognostic value compared to pT and TRG in patients treated with both chemoradiotherapy and perioperative chemotherapy.

A greater understanding of the relationship between the immune microenvironments of the tumour and lymph node will be useful in understanding the response to chemoradiotherapy and future immunotherapy. Harnessing the power of TLDNs may also boost the clinical outcomes following ICB. Indeed, ongoing clinical trials are investigating the role of ICB alongside neoadjuvant chemoradiotherapy in locally advanced, operable gastroesophageal adenocarcinoma (NCT02730546, NCT03044613) [31]. The LNME is suggested to be an immunosuppressive niche. One study using a mouse model of lung adenocarcinoma demonstrated that the LNME skews tumour antigen-specific $\mathrm{CD}^{+}$differentiation into regulatory $\mathrm{T}$ cells, thus promoting tumour immune escape [26]. In human melanoma patients, lymph node metastases are associated with the suppression of the CD1 $\mathrm{a}^{+} \mathrm{DC}$ subset, a cellular population that is efficient at cross-presenting neoantigens to $\mathrm{CD}^{+} \mathrm{T}$ cells. This immunosuppressive feature of the LNME was correlated to local tumour recurrence [5]. This also corresponded to an increased CD4:CD8 T cell ratio, and enrichment of regulatory $\mathrm{T}$ cells in the local nodal microenvironment in metastatic disease. This finding has since 
been replicated in breast and cervical cancer [24,36], where DC suppression was associated with impaired T cell effector function.

Our data is broadly in agreement with these studies; however, we not only analysed the immune profile of the LNME, but also compared it to the immune microenvironment of primary tumour samples, demonstrating that the TME exhibited greater immunosuppression compared to the LNME. We found that the TME had higher levels of infiltrating T cells co-expressing multiple ICs, which typically denote $\mathrm{T}$ cell exhaustion and functional impairment, compared to the LNME. The presence of IC expression by T cells in the LNME was previously reported in one study of cervical cancer patients, but it was not compared with the TME [24]. Using cytokine profiling, we also found that the TME had lower levels of IFN- $\gamma$, compared to the LNME. This suggests a more suppressive immune landscape, as IFN- $\gamma$ promotes DC antigen presentation as well as encouraging CD8 T cell responses [37]. Interestingly, in the KEYNOTE-028 clinical trial, an IFN- $\gamma$ gene expression signature was predictive of response to ICB in OC patients, underscoring its role in the immune response to OAC [38]. Levels of IL-9 and IL-27 were higher in the LNME compared to the TME, with both having mixed or tumour-promoting effects in terms of cancer progression [39]. Collectively, our findings indicate greater immunosuppression in the TME compared to the LNME, which could explain why intact TDLNs are indispensable for the clinical activity of ICB in mouse models $[27,28]$. However, the presence of exhausted T cells within a tumour does point toward a pre-existing antigen-specific anti-tumour immune response, a pre-requisite for response to ICBs.

The levels of immunosuppression in the LNME may also have clinical implications, as the presence of suppressed DC subsets in the TDLNs are negatively associated with recurrence and disease-free survival in melanoma and breast cancer [22,25]. Furthermore, PD-1, TIM-3, and TIGIT expression may represent mechanisms of immune escape in OAC and perhaps ICBs targeting PD-1, TIM-3, and TIGIT may represent a more effective and personalised rational approach for treating oesophagogastric cancer patients.

Although cancer cells were traditionally thought to spread to distant organs from lymph nodes through lymphatic vessels, recent preclinical studies suggest that cancer cells can invade local nodal blood vessels to enable a mixed haematogenous metastatic dissemination $[4,5]$. Thus, markers of angiogenesis and blood vessel formation may prove useful in understanding the metastatic potential of the TDLN. Levels of pro-angiogenic and wound healing markers including bFGF and PIGF were higher within the LNME than the TME. These mediators play important roles in promoting wound healing, angiogenesis, and tumour growth and therefore may play a role in remodelling the TDLN into a tumour permissive niche enabling nodal metastasis. The levels of the vascular damage protein bFGF were higher within the TME compared with the LNME. Known functions of bFGF include the enhancement of tumour cell proliferation, survival, motility, and wound healing [40]. Previous studies in oesophageal cancer demonstrated that bFGF overexpression is associated with a risk of tumour recurrence and reduced OS post-surgical resection, suggesting bFGF may play an important role in TME remodelling and enhancement of tumour progression [41,42].

Collectively, these data highlight a potentially detrimental role of tumour-promoting inflammation in OAC pathogenesis. Tumour-promoting inflammation is one of the enabling hallmarks of cancer and plays important roles in therapy resistance and tumour progression [43]. Interestingly, our study suggests that inflammation within the LNME may be beneficial as the levels of pro-inflammatory cytokines and chemokines are negatively correlated with advanced tumour stage and further supports the hypothesis of tumour-promoting inflammation. The TDLN is a key orchestrator of anti-tumour immunity; therefore, a heightened state of immune activity would be expected within the LNME. This also highlights that anti-cancer immune-promoting therapies should be developed to target the TDLN and not just the TME.

The role of Th17 cells in cancer is controversial, with studies suggesting both proand anti-tumour effects in diverse cancer types [44]. There is an abundance of work that 
detailed enrichment of Th17 cells in the TME of both gastric and oesophageal cancer [45,46], which still requires additional research to delineate the exact role of these cells in the TME and TDLN.

There are a number of limitations in this study in addition to the small cohort size for immunophenotyping, including the fact that fresh lymph node and tumour tissue samples were obtained at surgical resection, which is post-multimodal treatment. The impact of treatment can alter the microenvironment of the tumour and the lymph nodes within in the treatment field. However, understanding how treatment can affect the TME and LNME will have a significant bearing on the development of appropriate immunotherapeutic targeting. Whilst we have shown significant immunological differences between the tumour and the draining lymph node in this small cohort, additional larger studies using treatment naïve samples will be required to determine the real clinical significance of these potential therapeutic targets.

\section{Conclusions}

In summary, nodal involvement, and clinical and pathological tumour staging are significant prognostic indicators for OAC. These results also highlight the immunosuppressive nature of the TME demonstrated by higher percentages of $\mathrm{T}$ cells co-expressing multiple ICs in the TME compared to the LNME. The data are timely with the recently published Checkmate 577 trial advocating the use of immunotherapy in the adjuvant setting in carcinoma of the oeosphagogastric junction. Our data supports the hypothesis that combination therapeutic blockade of these aforementioned checkpoints may have a therapeutic rationale for boosting response rates in OAC.

Supplementary Materials: The following are available online at https:/ /www.mdpi.com/article/10 $.3390 /$ cancers13164005/s1, Figure S1: Chemokines, cytokines, Markers of angiogenesis and vascular injury. Table S1: Analyte in matched tumour and lymph node.

Author Contributions: All authors helped in the data analysis, preparation, and editing of this manuscript. N.E.D. performed experiments, statistical analysis of clinicopathological features, and writing of the manuscript; M.D. performed experiments, and contributed to writing of the manuscript and data interpretation; A.S. contributed to experiments; R.P. contributed to the writing of the manuscript; A.B.H. and F.O. contributed to the writing of the manuscript and data interpretation; R.K., C.H. and A.B. were responsible for clinical data collection; J.J.P. contributed to statistical analysis; A.B. was responsible for specimen and clinical data management; N.R. contributed to the structure of the manuscript and provision of specimens; C.L.D. contributed to study design; J.O., J.V.R. and J.L. contributed to the study concept, and design and editing of the manuscript; C.L.D., N.R. and J.V.R. provided specimens; J.O. contributed to the study design; and J.L. supervised, devised the original study, and drafted the manuscript. M.R.D. provided training in experimental techniques, assisted with clinical data acquisition and assisted in editing the manuscript. All authors have read and agreed to the published version of the manuscript.

Funding: This work was supported by the CROSS Cancer Charity (RCN: 15364).

Institutional Review Board Statement: The study was conducted according to the guidelines of the Declaration of Helsinki, and approved by the Institutional Ethics Committee of Tallaght University Hospital/St. James's Hospital (041113/10804 on 31 July 2019).

Informed Consent Statement: Informed consent was obtained from all subjects involved in the study.

Data Availability Statement: The data presented in this study are available in the article and Supplementary Material.

Acknowledgments: The authors wish to thank all patient volunteers at St James's Hospital Dublin.

Conflicts of Interest: The authors declare no conflict of interest. The funders had no role in the design of the study; in the collection, analyses, or interpretation of data; in the writing of the manuscript, or in the decision to publish the results. 


\section{References}

1. Yanagi, M.; Sasaki, K.; Uchikado, Y.; Omoto, I.; Arigami, T.; Kurahara, H.; Uenosono, Y.; Sakoda, M.; Maemura, K.; Natsugoe, S. Effect of Neoadjuvant Chemoradiotherapy on Lymph Node Micrometastases in Thoracic Esophageal Cancer. Anticancer Res. 2018, 38, 893-900. [PubMed]

2. Kelsen, D.P.; Ginsberg, R.; Pajak, T.F.; Sheahan, D.G.; Gunderson, L.; Mortimer, J.; Estes, N.; Haller, D.G.; Ajani, J.; Kocha, W.; et al. Chemotherapy followed by surgery compared with surgery alone for localized esophageal cancer. N. Engl. J. Med. 1998, 339, 1979-1984. [CrossRef]

3. Korst, R.J.; Kansler, A.L.; Port, J.L.; Lee, P.C.; Kerem, Y.; Altorki, N.K. Downstaging of T or N Predicts, Long-Term, Survival After, Preoperative Chemotherapy and Radical, Resection for Esophageal, Carcinoma. Ann. Thorac. Surg. 2006, 82, 480-485. [CrossRef] [PubMed]

4. Brown, M.; Assen, F.P.; Leithner, A.; Abe, J.; Schachner, H.; Asfour, G.; Bago-Horvath, Z.; Stein, J.V.; Uhrin, P.; Sixt, M.; et al. Lymph node blood vessels provide exit routes for metastatic tumor cell dissemination in mice. Science 2018, 359, 1408. [CrossRef]

5. Pereira, E.R.; Kedrin, D.; Seano, G.; Gautier, O.; Meijer, E.F.J.; Jones, D.; Chin, S.-M.; Kitahara, S.; Bouta, E.M.; Chang, J.; et al. Lymph node metastases can invade local blood vessels, exit the node, and colonize distant organs in mice. Science 2018, $359,1403$. [CrossRef]

6. Halsted, W.S. The results of radical operations for the cure of carcinoma of the breast. Ann. Surg. 1907, 46, 1-19. [CrossRef]

7. $\quad$ Rice, T.W.; Ishwaran, H.; Hofstetter, W.L.; Schipper, P.H.; Kesler, K.A.; Law, S.; Lerut, T.; Denlinger, C.E.; Salo, J.A.; Scott, W.J.; et al. Esophageal, Cancer: Associations, With (pN+) Lymph, Node Metastases. Ann. Surg. 2017, 265, 122-129. [CrossRef] [PubMed]

8. Peyre, C.G.; Hagen, J.A.; DeMeester, S.R.; Altorki, N.K.; Ancona, E.; Griffin, S.M.; Holscher, A.; Lerut, T.; Law, S.; Rice, T.W.; et al. The number of lymph nodes removed predicts survival in esophageal cancer: An international study on the impact of extent of surgical resection. Ann. Surg. 2008, 248, 549-556. [CrossRef]

9. Mariette, C.; Piessen, G.; Briez, N.; Triboulet, J.P. The number of metastatic lymph nodes and the ratio between metastatic and examined lymph nodes are independent prognostic factors in esophageal cancer regardless of neoadjuvant chemoradiation or lymphadenectomy extent. Ann. Surg. 2008, 247, 365-371. [CrossRef]

10. Barbour, A.P.; Jones, M.; Gonen, M.; Gotley, D.C.; Thomas, J.; Thomson, D.B.; Burmeister, B.; Smithers, B.M. Refining esophageal cancer staging after neoadjuvant therapy: Importance of treatment response. Ann. Surg. Oncol. 2008, 15, 2894-2902. [CrossRef]

11. Donohoe, C.L.; O’Farrell, N.J.; Grant, T.; King, S.; Clarke, L.; Muldoon, C.; Reynolds, J.V. Classification of Pathologic, Response to Neoadjuvant, Therapy in Esophageal and Junctional, Cancer: Assessment of Existing, Measures and Proposal of a Novel 3-Point, Standard. Ann. Surg. 2013, 258, 784-792. [CrossRef] [PubMed]

12. Hölscher, A.H.; Drebber, U.; Schmidt, H.; Bollschweiler, E. Prognostic classification of histopathologic response to neoadjuvant therapy in esophageal adenocarcinoma. Ann. Surg. 2014, 260, 779-784; discussion 784-785. [CrossRef]

13. Mandard, A.M.; Dalibard, F.; Mandard, J.C.; Marnay, J.; Henry-Amar, M.; Petiot, J.F.; Roussel, A.; Jacob, J.H.; Segol, P.; Samama, G.; et al. Pathologic assessment of tumor regression after preoperative chemoradiotherapy of esophageal carcinoma. Clinicopathologic correlations. Cancer 1994, 73, 2680-2686. [CrossRef]

14. Noble, F.; Nolan, L.; Bateman, A.C.; Byrne, J.P.; Kelly, J.J.; Bailey, I.S.; Sharland, D.M.; Rees, C.N.; Iveson, T.J.; Underwood, T.J.; et al. Refining pathological evaluation of neoadjuvant therapy for adenocarcinoma of the esophagus. World J. Gastroenterol. 2013, 19, 9282-9293. [CrossRef] [PubMed]

15. Noble, F.; Lloyd, M.A.; Turkington, R.; Griffiths, E.; O’Donovan, M.; O’Neill, J.R.; Mercer, S.; Parsons, S.L.; Fitzgerald, R.C.; Underwood, T.J.; et al. Multicentre cohort study to define and validate pathological assessment of response to neoadjuvant therapy in oesophagogastric adenocarcinoma. BJS (Br. J. Surg.) 2017, 104, 1816-1828. [CrossRef]

16. Smyth, E.C.; Fassan, M.; Cunningham, D.; Allum, W.H.; Okines, A.F.; Lampis, A.; Hahne, J.C.; Rugge, M.; Peckitt, C.; Nankivell, M.; et al. Effect of Pathologic, Tumor Response and Nodal, Status on Survival in the Medical, Research Council, Adjuvant Gastric, Infusional Chemotherapy, Trial. J. Clin. Oncol. 2016, 34, 2721-2727. [CrossRef] [PubMed]

17. Zanoni, A.; Verlato, G.; Giacopuzzi, S.; Motton, M.; Casella, F.; Weindelmayer, J.; Ambrosi, E.; Leo, A.D.; Vassiliadis, A.; Ricci, F.; et al. ypN0: Does, It Matter, How You, Get There? Nodal, Downstaging in Esophageal, Cancer. Ann. Surg. Oncol. 2016, 23 (Suppl. 5), 998-1004. [CrossRef]

18. Shapiro, J.; Biermann, K.; van Klaveren, D.; Offerhaus, G.J.A.; ten Kate, F.J.W.; Meijer, S.L.; van Berge Henegouwen, M.I.; Steyerberg, E.W.; Wijnhoven, B.P.L.; van Lanschot, J.J.B. Prognostic, Value of Pretreatment, Pathological Tumor, Extent in Patients, Treated With, Neoadjuvant Chemoradiotherapy, Plus Surgery for Esophageal or Junctional, Cancer. Ann. Surg. 2017, 265, 356-362. [CrossRef]

19. Rotman, J.; Koster, B.D.; Jordanova, E.S.; Heeren, A.M.; de Gruijl, T.D. Unlocking the therapeutic potential of primary tumordraining lymph nodes. Cancer Immunol. Immunother. 2019, 68, 1681-1688. [CrossRef]

20. Leach, D.R.; Krummel, M.F.; Allison, J.P. Enhancement of antitumor immunity by CTLA-4 blockade. Science 1996, 271, 1734-1736. [CrossRef]

21. Chen, D.S.; Mellman, I. Oncology meets immunology: The cancer-immunity cycle. Immunity 2013, 39, 1-10. [CrossRef]

22. van Pul, K.M.; Vuylsteke, R.J.C.L.M.; van de Ven, R.; te Velde, E.A.; Rutgers, E.J.T.; van den Tol, P.M.; Stockmann, H.B.A.C.; de Gruijl, T.D. Selectively hampered activation of lymph node-resident dendritic cells precedes profound T cell suppression and metastatic spread in the breast cancer sentinel lymph node. J. Immunother. Cancer 2019, 7, 133. [CrossRef] 
23. van de Ven, R.; van den Hout, M.F.; Lindenberg, J.J.; Sluijter, B.J.; van Leeuwen, P.A.; Lougheed, S.M.; Meijer, S.; van den Tol, M.P.; Scheper, R.J.; de Gruijl, T.D. Characterization of four conventional dendritic cell subsets in human skin-draining lymph nodes in relation to T-cell activation. Blood 2011, 118, 2502-2510. [CrossRef] [PubMed]

24. Heeren, A.M.; Koster, B.D.; Samuels, S.; Ferns, D.M.; Chondronasiou, D.; Kenter, G.G.; Jordanova, E.S.; de Gruijl, T.D. High and interrelated rates of PD-L1+CD14+ antigen-presenting cells and regulatory T cells mark the microenvironment of metastatic lymph nodes from patients with cervical cancer. Cancer Immunol. Res. 2015, 3, 48-58. [CrossRef] [PubMed]

25. van den Hout, M.; Koster, B.D.; Sluijter, B.J.R.; Molenkamp, B.G.; van de Ven, R.; van den Eertwegh, A.J.M.; Scheper, R.J.; van Leeuwen, P.A.M.; van den Tol, M.P.; de Gruijl, T.D. Melanoma, Sequentially Suppresses, Different DC Subsets in the Sentinel, Lymph Node, Affecting, Disease Spread and Recurrence. Cancer Immunol. Res. 2017, 5, 969-977. [CrossRef] [PubMed]

26. Alonso, R.; Flament, H.; Lemoine, S.; Sedlik, C.; Bottasso, E.; Péguillet, I.; Prémel, V.; Denizeau, J.; Salou, M.; Darbois, A.; et al. Induction of anergic or regulatory tumor-specific CD4+ T cells in the tumor-draining lymph node. Nat. Commun. 2018, 9, 2113. [CrossRef] [PubMed]

27. Fransen, M.F.; Schoonderwoerd, M.; Knopf, P.; Camps, M.G.M.; Hawinkels, L.J.A.C.; Kneilling, M.; van Hall, T.; Ossendorp, F. Tumor-draining lymph nodes are pivotal in PD-1/PD-L1 checkpoint therapy. JCI Insight 2018, 3, e124507. [CrossRef]

28. Chamoto, K.; Chowdhury, P.S.; Kumar, A.; Sonomura, K.; Matsuda, F.; Fagarasan, S.; Honjo, T. Mitochondrial activation chemicals synergize with surface receptor PD-1 blockade for T cell-dependent antitumor activity. Proc. Natl. Acad. Sci. USA 2017, 114, E761-E770. [CrossRef]

29. Marciscano, A.E.; Ghasemzadeh, A.; Nirschl, T.R.; Theodros, D.; Kochel, C.M.; Francica, B.J.; Muroyama, Y.; Anders, R.A.; Sharabi, A.B.; Velarde, E.; et al. Elective, Nodal Irradiation, Attenuates the Combinatorial, Efficacy of Stereotactic, Radiation Therapy and Immunotherapy. Clin. Cancer Res. 2018, 24, 5058-5071. [CrossRef]

30. Hebb, J.P.O.; Mosley, A.R.; Vences-Catalán, F.; Rajasekaran, N.; Rosén, A.; Ellmark, P.; Felsher, D.W. Administration of low-dose combination anti-CTLA4, anti-CD137, and anti-OX40 into murine tumor or proximal to the tumor draining lymph node induces systemic tumor regression. Cancer Immunol. Immunother. 2018, 67, 47-60. [CrossRef]

31. Power, R.; Lowery, M.A.; Reynolds, J.V.; Dunne, M.R. The, Cancer-Immune, Set Point in Oesophageal, Cancer. Front. Oncol. 2020, 10, 891. [CrossRef] [PubMed]

32. Donlon, N.E.; Power, R.; Hayes, C.; Reynolds, J.V.; Lysaght, J. Radiotherapy, immunotherapy, and the tumour microenvironment: Turning an immunosuppressive milieu into a therapeutic opportunity. Cancer Lett. 2021, 502, 84-96. [CrossRef]

33. Donlon, N.E.; Power, R.; Hayes, C.; Davern, M.; Reynolds, J.V.; Lysaght, J. Radiation and Immunotherapy in Upper, Gastrointestinal Cancers: The, Current State of Play. Int. J. Mol. Sci. 2021, 22, 1071. [CrossRef]

34. Donlon, N.E.; Elliott, J.A.; Donohoe, C.L.; Murphy, C.F.; Nugent, T.; Moran, B.; King, S.; Ravi, N.; Reynolds, J.V. Adverse, Biology in Adenocarcinoma of the Esophagus and Esophagogastric, Junction Impacts, Survival and Response to Neoadjuvant, Therapy Independent of Anatomic, Subtype. Ann. Surg. 2020, 272, 814-819. [CrossRef]

35. Rahman, S.A.; Walker, R.C.; Lloyd, M.A.; Grace, B.L.; van Boxel, G.I.; Kingma, B.F.; Ruurda, J.P.; van Hillegersberg, R.; Harris, S.; Parsons, S.; et al. Machine learning to predict early recurrence after oesophageal cancer surgery. Br. J. Surg. 2020, 107, 1042-1052. [CrossRef]

36. Kim, R.; Kawai, A.; Wakisaka, M.; Funaoka, Y.; Tasaka, Y.; Yasuda, N.; Hidaka, M.; Morita, Y.; Ohtani, S.; Ito, M.; et al. Immune response induced by preoperative chemotherapy in breast cancer: Role of peripheral natural killer (pNK) cell activity, tumorinfiltrating lymphocytes (TILs), and tumor microenvironment factors (TMEFs). J. Clin. Oncol. 2018, 36 (Suppl. 15), e12644. [CrossRef]

37. van Sandick, J.W.; Boermeester, M.A.; Gisbertz, S.S.; ten Berge, I.J.; Out, T.A.; van der Pouw Kraan, T.C.; van Lanschot, J.J. Lymphocyte subsets and $\mathrm{T}(\mathrm{h}) 1 / \mathrm{T}(\mathrm{h}) 2$ immune responses in patients with adenocarcinoma of the oesophagus or oesophagogastric junction: Relation to pTNM stage and clinical outcome. Cancer Immunol. Immunother. 2003, 52, 617-624. [CrossRef]

38. Doi, T.; Piha-Paul, S.A.; Jalal, S.I.; Saraf, S.; Lunceford, J.; Koshiji, M.; Bennouna, J. Safety and Antitumor, Activity of the Anti-Programmed, Death-1 Antibody, Pembrolizumab in Patients, With Advanced, Esophageal Carcinoma. J. Clin. Oncol. 2017, 36, 61-67. [CrossRef] [PubMed]

39. Nagarsheth, N.; Wicha, M.S.; Zou, W. Chemokines in the cancer microenvironment and their relevance in cancer immunotherapy. Nat. Rev. Immunol. 2017, 17, 559-572. [CrossRef] [PubMed]

40. Paris, F.; Fuks, Z.; Kang, A.; Capodieci, P.; Juan, G.; Ehleiter, D.; Haimovitz-Friedman, A.; Cordon-Cardo, C.; Kolesnick, R. Endothelial apoptosis as the primary lesion initiating intestinal radiation damage in mice. Science 2001, 293, 293-297. [CrossRef] [PubMed]

41. Barclay, C.; Li, A.W.; Geldenhuys, L.; Baguma-Nibasheka, M.; Porter, G.A.; Veugelers, P.J.; Murphy, P.R.; Casson, A.G. Basic fibroblast growth factor (FGF-2) overexpression is a risk factor for esophageal cancer recurrence and reduced survival, which is ameliorated by coexpression of the FGF-2 antisense gene. Clin. Cancer Res. 2005, 11, 7683-7691. [CrossRef] [PubMed]

42. Donlon, N.E.; Sheppard, A.; Davern, M.; O'Connell, F.; Phelan, J.J.; Power, R.; Nugent, T.; Dinneen, K.; Aird, J.; Greene, J.; et al. Linking Circulating Serum Proteins with Clinical Outcomes in Esophageal Adenocarcinoma-An Emerging Role for Chemokines. Cancers 2020, 12, 3356. [CrossRef]

43. Buckley, A.M.; Lynam-Lennon, N.; O'Neill, H.; O'Sullivan, J. Targeting hallmarks of cancer to enhance radiosensitivity in gastrointestinal cancers. Nat. Rev. Gastroenterol. Hepatol. 2020, 17, 298-313. [CrossRef] 
44. Bailey, S.R.; Nelson, M.H.; Himes, R.A.; Li, Z.; Mehrotra, S.; Paulos, C.M. Th17 Cells in Cancer: The, Ultimate Identity, Crisis. Front. Immunol. 2014, 5, 276. [CrossRef]

45. Chen, D.; Hu, Q.; Mao, C.; Jiao, Z.; Wang, S.; Yu, L.; Xu, Y.; Dai, D.; Yin, L.; Xu, H. Increased, IL-17-producing CD4(+) T cells in patients with esophageal cancer. Cell. Immunol. 2012, 272, 166-174. [CrossRef] [PubMed]

46. Pinchuk, I.V.; Morris, K.T.; Nofchissey, R.A.; Earley, R.B.; Wu, J.-Y.; Ma, T.Y.; Beswick, E.J. Stromal cells induce Th17 during Helicobacter pylori infection and in the gastric tumor microenvironment. PLoS ONE 2013, 8, e53798. [CrossRef] [PubMed] 